\title{
The variational problem of fractional-order control systems
}

\author{
Biao Liu*, Yanhua Wen and Xian-feng Zhou
}

${ }^{\text {"Correspondence: }}$

liubia00807@126.com

School of Mathematical Sciences,

Anhui University, Hefei, 230039,

China

\begin{abstract}
This article discusses the control system of fractional endpoint variable variational problems. For this problem, we prove the Euler-Lagrange type necessary conditions which must be satisfied for the given functional to be extremum. Finally, one example is provided to show the application of our results.
\end{abstract}

Keywords: variational problem; necessary condition; fractional-order control systems

\section{Introduction}

Fractional calculus is an old mathematical topic since the seventeenth century, yet it has only received much attention and interest in the past 20 years. For more details on the basic theory of fractional calculus, one can see the monographs [1-4] and the references therein.

As one of the important topics in control theory, the variational method plays an important role in the analysis control systems and is an important branch of mathematics study functional extremum. In recent years, the variational method is widely used in physics, economics, electrical engineering, image processing fields, etc.

Moreover, the numerical methods for solving fractional differential equations, optimal control and variational problems have a good development. In [5], Bhrawy et al. investigated a new spectral collocation scheme, which obtained a numerical solution of this equation with variable coefficients on a semi-infinite domain. In [6], Doha et al. introduced a numerical technique for solving a general form of the fractional optimal control problem. And in [7], Bhrawy et al. used the Rayleigh-Ritz method for the necessary conditions of optimization and the operational matrix of fractional derivatives together with the help of the properties of the shifted Legendre orthonormal polynomials to reduce the fractional optimal control problem to solving a system of algebraic equations that greatly simplifies the problem. In [8], Bhrawy and Zaky proposed and analyzed an efficient operational formulation of spectral tau method for a multi-term time-space fractional differential equation with Dirichlet boundary conditions. In [9], based on the shifted Legendre orthonormal polynomials, Ezz-Eldien et al. employed the operational matrix of fractional derivatives, the Legendre-Gauss quadrature formula and the Lagrange multiplier method for reducing such a problem into a problem consisting of solving a system of algebraic equations.

0 2015 Liu et al.: licensee Springer. This is an Open Access article distributed under the terms of the Creative Commons Attribution License (http://creativecommons.org/licenses/by/4.0), which permits unrestricted use, distribution, and reproduction in any medium, provided the original work is properly credited. 
However, at present, very little work has been done in the area of fractional calculus of variations [10-13]. In [10], Jiao and Zhou used the critical point theory to solve the existence of solutions for a class of fractional boundary value problems. In [12, 13], the author presented a new approach to mechanics that allows one to obtain the equations for a nonconservative system using certain functionals. In [11], the author proposed the fractional-order variational problem and gave solution for a class of variational problems with fixed boundary value

$$
J[x]=\int_{t_{0}}^{t_{f}} F\left(t, x, D_{t_{0}, t}^{\alpha} x, D_{t, t_{f}}^{\beta} x\right) d t
$$

and the boundary conditions $x\left(t_{0}\right)=x_{0}, x\left(t_{f}\right)=x_{f}$ are fixed. But [11] did not discuss the problem of variable boundary conditions and transversal conditions. In other words, the boundary condition $x\left(t_{0}\right)=x_{0}$ is fixed, but $x\left(t_{f}\right)=C\left(t_{f}\right)$ is variable, or vice versa. Inspired by the above-mentioned works, in this paper, we follow the ideas to investigate the optimality of control systems.

This paper is organized as follows. In Section 2, we briefly review the definitions of Riemann-Liouville fractional integrals and derivatives and some lemmas. In Section 3, we give the necessary conditions for the fractional-order functional variational problem with fixed and variable boundary. In Section 4, we give an example to show the application of our results, and Section 5 briefly summarizes the results of this paper and future work.

\section{Preliminaries and lemmas}

In this section, we give some basic definitions and results that are used throughout this paper. For more details, please see [1-4].

Definition 2.1 [1-4] Let $[a, b]$ be a finite interval on the real axis $R$. The RiemannLiouville fractional integrals $I_{a, t}^{\alpha} f$ and $I_{t, b}^{\alpha} f$ of order $\alpha>0$ are defined by

$$
I_{a, t}^{\alpha} f(t)=\frac{1}{\Gamma(\alpha)} \int_{a}^{t} \frac{f(\tau)}{(t-\tau)^{1-\alpha}} d \tau, \quad t>a
$$

and

$$
I_{t, b}^{\alpha} f(t)=\frac{1}{\Gamma(\alpha)} \int_{t}^{b} \frac{f(\tau)}{(t-\tau)^{1-\alpha}} d \tau, \quad t<b
$$

respectively. Here, $\Gamma(\cdot)$ denotes the gamma function. These integrals are called the leftsided and the right-sided fractional integrals.

Definition $2.2[1-4]$ The Riemann-Liouville fraction derivatives $D_{a, t}^{\alpha} y$ and $D_{t, b}^{\alpha} y$ of order $\alpha>0$ are defined by

$$
\begin{aligned}
D_{a, t}^{\alpha} y(t) & =\left(\frac{d}{d t}\right)^{n}\left(I_{a, t}^{n-\alpha} y(t)\right) \\
& =\frac{1}{\Gamma(n-\alpha)}\left(\frac{d}{d t}\right)^{n} \int_{a}^{t} \frac{f(\tau)}{(t-\tau)^{\alpha-n+1}} d \tau \quad(n=[\alpha]+1, t>a)
\end{aligned}
$$


and

$$
\begin{aligned}
D_{t, b}^{\alpha} y(t) & =\left(\frac{d}{d t}\right)^{n}\left(I_{t, b}^{n-\alpha} y(t)\right) \\
& =\frac{1}{\Gamma(n-\alpha)}\left(\frac{d}{d t}\right)^{n} \int_{t}^{b} \frac{f(\tau)}{(t-\tau)^{\alpha-n+1}} d \tau \quad(n=[\alpha]+1, x<b),
\end{aligned}
$$

respectively, where $[\alpha]$ means the integral part of $\alpha$.

Remark 2.1 [1-4] Obviously, if $0<\alpha<1$, then

$$
D_{a, t}^{\alpha} y(t)=\frac{1}{\Gamma(1-\alpha)} \frac{d}{d t} \int_{a}^{t} \frac{f(\tau)}{(t-\tau)^{\alpha}} d \tau \quad(0<\alpha<1, x>a)
$$

and

$$
D_{t, b}^{\alpha} y(t)=\frac{1}{\Gamma(1-\alpha)} \frac{d}{d t} \int_{t}^{b} \frac{f(\tau)}{(t-\tau)^{\alpha}} d \tau \quad(0<\alpha<1, x<b) .
$$

Lemma 2.1 (Fractional integration by parts [1]) Assume that $D_{a, t}^{\alpha} f(x)$ and $D_{t, b}^{\alpha} g(x)$ are existent and continuous on $[a, b]$. Let $f(t)$ and $g(t)$ be two continuous functions defined on $t \in[a, b], \alpha>0$, and $f(t)$ or $g(t)$ and their until $m$ derivatives are zero at $t=a, b$, and $m$ is less than the largest integer of $\alpha$. Then

(i)

$$
\int_{a}^{b} \varphi(t)\left(I_{a, t}^{\alpha} \psi(t)\right) d t=\int_{a}^{b} \psi(t)\left(I_{t, b}^{\alpha} \varphi(t)\right) d t
$$

(ii)

$$
\int_{a}^{b}\left(D_{a, t}^{\alpha} f(t)\right) g(t) d t=\int_{a}^{b} f(t)\left(D_{t, b}^{\alpha} g(t)\right) d t .
$$

\section{The fractional variational problems-variable endpoints}

Definition 3.1 Assume that $F=F\left(t, x, D_{t_{0}, t^{x}}^{\alpha} x, D_{t, t_{f}}^{\beta} x\right)$ is a function with continuous first and second (partial) derivatives with respect to all its arguments. Then, among all functions $x(t)$ which have continuous LRLFD of order $\alpha$ and RRLFD of order $\beta$ for $t_{0} \leq t \leq t_{f}$ and satisfy the boundary conditions

$$
x\left(t_{0}\right)=x_{0}, \quad x\left(t_{f}\right)=C\left(t_{f}\right) .
$$

Looking for a function $x(t)$ such that the functional

$$
J(x)=\int_{t_{0}}^{t_{f}} F\left(t, x, D_{t_{0}, t}^{\alpha} x, D_{t_{0}, t_{f}}^{\beta} x\right) d t
$$

has extreme value, where $0<\alpha, \beta \leq 1$ and the endpoint $C\left(t_{f}\right)$ is variable.

Theorem 3.1 Let $J(x)$ be a functional of the form

$$
J(x)=\int_{t_{0}}^{t_{f}} F\left(t, x, D_{t_{0}, t}^{\alpha} x, D_{t, t_{f}}^{\beta} x\right) d t
$$


defined on the set of functions $x(t)$ which have continuous LRLFD of order $\alpha$ and RRLFD of order $\beta$ in $\left[t_{0}, t_{f}\right]$ and satisfy the boundary conditions $x\left(t_{0}\right)=x_{0}$ and $x\left(t_{f}\right)=C\left(t_{f}\right)$. Then a necessary condition for $J[x]$ to have an extremum for a given function $x(t)$ is that $x(t)$ satisfies the following Euler-Lagrange equation and terminal transversality condition:

$$
\begin{aligned}
& \frac{\partial F}{\partial x}-D_{t, t_{f}}^{\alpha} \frac{\partial F}{\partial D_{t_{0}, t}^{\alpha} x}-D_{t_{0}, t}^{\beta} \frac{\partial F}{\partial D_{t, t_{f}}^{\beta} x}=0, \\
& \left.\left(\frac{\partial F}{\partial D_{t_{0}, t}^{\alpha} x} D_{t_{0}, t}^{\alpha}(C-x)+\frac{\partial F}{\partial D_{t, t_{f}}^{\beta} x} D_{t, t_{f}}^{\beta}(C-x)+F\right)\right|_{t=t_{f}}=0 .
\end{aligned}
$$

Proof To prove the necessary conditions for the extremum, assume that $x^{*}(t)$ is the desired function. Let $\varepsilon \in R$, and define a family of curves

$$
x(t)=x^{*}(t)+\varepsilon \eta(t)
$$

where $\eta(t)$ is a continuous differentiable function for all given, which satisfy the boundary conditions, i.e.,

$$
\eta\left(t_{0}\right)=0
$$

Due to the changing terminal time $t_{f}$, each has its own trajectory terminal point $t_{f}$. Therefore we must define a terminal times set corresponding to $x(t)$,

$$
t_{f}=t_{f}^{*}+\varepsilon \xi\left(t_{f}\right)
$$

Since $D_{a, t}^{\alpha}$ and $D_{t, t_{f}}^{\beta}$ are linear operations, it follows that

$$
\begin{aligned}
& D_{t_{0}, t}^{\alpha} x(t)=D_{t_{0}, t}^{\alpha} x^{*}(t)+\varepsilon D_{t_{0}, t}^{\alpha} \eta(t), \\
& D_{t, t_{f}}^{\beta} x(t)=D_{t, t_{f}}^{\beta} x^{*}(t)+\varepsilon D_{t, t_{f}}^{\beta} \eta(t) .
\end{aligned}
$$

Substituting Eqs. (3.6) and (3.8)-(3.10) into Eq. (3.3),

$$
\begin{aligned}
J(\varepsilon)= & \int_{t_{0}}^{t_{f}^{*}+\varepsilon \xi\left(t_{f}\right)} F\left(t, x^{*}+\varepsilon \eta, D_{t_{0}, t}^{\alpha} x^{*}+\varepsilon D_{t_{0}, t}^{\alpha} \eta, D_{t, t_{f}}^{\beta} x^{*}+\varepsilon D_{t_{0}, t}^{\beta} \eta\right) d t \\
= & \int_{t_{0}}^{t_{f}^{*}} F\left(t, x^{*}+\varepsilon \eta, D_{t_{0}, x^{2}}^{\alpha} x^{*}+\varepsilon D_{t_{0}, t}^{\alpha} \eta, D_{t, t_{f}}^{\beta} x^{*}+\varepsilon D_{t_{0}, t}^{\beta} \eta\right) d t \\
& +\int_{t_{f}^{*}}^{t_{f}^{*}+\varepsilon \xi\left(t_{f}\right)} F\left(t, x^{*}+\varepsilon \eta, D_{t_{0}, t^{*}}^{\alpha} x^{*}+\varepsilon D_{t_{0}, t}^{\alpha} \eta, D_{t, t_{f}}^{\beta} x^{*}+\varepsilon D_{t_{0}, t}^{\beta} \eta\right) d t \\
\approx & \int_{t_{0}}^{t_{f}^{*}} F\left(t, x^{*}+\varepsilon \eta, D_{a, t}^{\alpha} x^{*}+\varepsilon D_{t_{0}, t}^{\alpha} \eta, D_{t, t_{f}}^{\beta} x^{*}+\varepsilon D_{t_{0}, t}^{\beta} \eta\right) d t \\
& +\varepsilon \xi\left(t_{f}\right) F\left(t_{f}^{*}, x^{*}\left(t_{f}^{*}\right), D_{t_{0}, t^{2}}^{\alpha} x^{*}\left(t_{f}^{*}\right), D_{t, t_{f}}^{\beta} x^{*}\left(t_{f}^{*}\right)\right)
\end{aligned}
$$

we find that for each $\eta(t)$ there is only just a function of $\varepsilon$. Note that $J(\varepsilon)$ is extremum at $\varepsilon=0$, and the differential of Eq. (3.11) with respect to $\varepsilon$, we obtain

$$
\left.\frac{\partial J}{\partial \varepsilon}\right|_{\varepsilon=0}=\int_{t_{0}}^{t_{f}^{*}}\left(\frac{\partial F}{\partial x} \eta+\frac{\partial F}{\partial D_{t_{0}, t}^{\alpha} x} D_{t_{0}, t}^{\alpha} \eta+\frac{\partial F}{\partial D_{t, t_{f}}^{\beta} x} D_{t, t_{f}}^{\beta} \eta\right) d t+\left.\xi\left(t_{f}\right) F\right|_{t_{f}^{*}} .
$$


Equation (3.12) is also called the variations of $J(x)$ at $x(t)$ along $\eta(t)$. A necessary condition for $J(\varepsilon)$ to have an extremum is that $\left.\frac{\partial J}{\partial \varepsilon}\right|_{\varepsilon=0}=0$, and it is true for any admissible $\eta(t)$. This leads to the condition that for $J(x)$ to have an extremum for $x=x^{*}(t)$,

$$
\int_{t_{0}}^{t_{f}^{*}}\left(\frac{\partial F}{\partial x} \eta+\frac{\partial F}{\partial D_{t_{0}, t}^{\alpha} x} D_{t_{0}, t}^{\alpha} \eta+\frac{\partial F}{\partial D_{t, t_{f}}^{\beta} x} D_{t, t_{f}}^{\beta} \eta\right) d t+\left.\xi\left(t_{f}\right) F\right|_{t_{f}^{*}}=0
$$

for all admissible $\eta(t)$. Using the definition of fractional derivatives and the formula for fractional integration by parts, the second and third integral in Eq. (3.13) can be written as

$$
\begin{aligned}
& \int_{t_{0}}^{t_{f}^{*}} \frac{\partial F}{\partial D_{t_{0}, t}^{\alpha} x(t)} D_{t_{0}, t}^{\alpha} \eta(t) d t \\
& =\left.\frac{\partial F}{\partial D_{t_{0}, t}^{\alpha} x(t)} I_{t_{0}, t}^{1-\alpha} \eta(t)\right|_{t_{0}} ^{t_{f}}-\int_{t_{0}}^{t_{f}} \frac{d}{d t}\left(\frac{\partial F}{\partial D_{t_{0}, t}^{\alpha} x(t)}\right) I_{t_{0}, t}^{1-\alpha} \eta(t) d t \\
& =\left.\frac{\partial F}{\partial D_{t_{0}, t}^{\alpha} x(t)} I_{t_{0}, t}^{1-\alpha} \eta(t)\right|_{t_{0}} ^{t_{f}}-\int_{t_{0}}^{t_{f}} \eta(t) D_{t, t_{f}}^{\alpha}\left(\frac{\partial F}{\partial D_{t_{0}, t}^{\alpha} x(t)}\right) d t, \\
& \int_{t_{0}}^{t_{f}^{*}} \frac{\partial F}{\partial D_{t, t_{f}}^{\beta} x(t)} D_{t, t_{f}}^{\beta} \eta(t) d t \\
& =\left.\frac{\partial F}{\partial D_{t, t_{f}}^{\beta} x(t)} I_{t, t_{f}}^{1-\beta} \eta(t)\right|_{t_{0}} ^{t_{f}}-\int_{t_{0}}^{t_{f}} \frac{d}{d t}\left(\frac{\partial F}{\partial D_{t_{0}, t}^{\beta} x(t)}\right) I_{t_{0}, t}^{1-\beta} \eta(t) d t \\
& =\left.\frac{\partial F}{\partial D_{t, t_{f}}^{\beta} x(t)} I_{t, t_{f}}^{1-\beta} \eta(t)\right|_{t_{0}} ^{t_{f}}-\int_{t_{0}}^{t_{f}} \eta(t) D_{t_{0}, t}^{\beta}\left(\frac{\partial F}{\partial D_{t, t_{f}}^{\beta} x(t)}\right) d t .
\end{aligned}
$$

Substituting Eqs. (3.14) and (3.15) into Eq. (3.13) yields

$$
\begin{aligned}
& \int_{t_{0}}^{t_{f}^{*}}\left\{\frac{\partial F}{\partial x}-D_{t, t_{f}}^{\alpha}\left(\frac{\partial F}{\partial D_{t_{0}, t}^{\alpha} x}\right)-D_{t_{0}, t}^{\beta}\left(\frac{\partial F}{\partial D_{t, t_{f}}^{\beta} x}\right)\right\} \eta d t \\
& +\left.\left(\frac{\partial F}{\partial D_{t_{0}, t}^{\alpha}} I_{t_{0}, t}^{1-\alpha} \eta+\frac{\partial F}{\partial D_{t, t_{f}}^{\beta} x} I_{t, t_{f}}^{1-\beta} \eta\right)\right|_{t_{0}} ^{t_{f}}+\left.\xi\left(t_{f}\right) F\right|_{t_{f}^{*}}=0 .
\end{aligned}
$$

In virtue of $\eta\left(t_{0}\right)=0$, Eq. (3.16) can be written in the form

$$
\begin{gathered}
\int_{t_{0}}^{t_{f}^{*}}\left\{\frac{\partial F}{\partial x}-D_{t, t_{f}}^{\alpha}\left(\frac{\partial F}{\partial D_{t_{0}, t}^{\alpha} x}\right)-D_{t_{0}, t}^{\beta}\left(\frac{\partial F}{\partial D_{t, t_{f}}^{\beta} x}\right)\right\} \eta d t \\
+\left.\left(\frac{\partial F}{\partial D_{t_{0}, t}^{\alpha} x} I_{t_{0}, t}^{1-\alpha} \eta+\frac{\partial F}{\partial D_{t, t_{f}}^{\beta} x} I_{t, t_{f}}^{1-\beta} \eta+\xi\left(t_{f}\right) F\right)\right|_{t_{f}^{*}}=0 .
\end{gathered}
$$

Note that $\eta\left(t_{f}^{*}\right)$ and $\xi\left(t_{f}\right)$ are not independent of each other, they are affected by the terminal constraint $\left.x(t)\right|_{t=t_{f}}=C\left(t_{f}\right)$, namely

$$
x\left(t_{f}^{*}+\varepsilon \xi\left(t_{f}\right)\right)+\varepsilon \eta\left(t_{f}^{*}+\varepsilon \xi\left(t_{f}\right)\right)=C\left(t_{f}^{*}+\varepsilon \xi\left(t_{f}\right)\right) .
$$


Differentiating Eq. (3.18) with respect to $\varepsilon$ and letting $\varepsilon \rightarrow 0$, we have

$$
\xi\left(t_{f}\right) \dot{x}\left(t_{f}^{*}\right)+\eta\left(t_{f}^{*}\right)=\xi\left(t_{f}\right) \dot{C}\left(t_{f}^{*}\right) .
$$

That is,

$$
\eta\left(t_{f}^{*}\right)=\xi\left(t_{f}\right)\left[\dot{C}\left(t_{f}^{*}\right)-\dot{x}\left(t_{f}^{*}\right)\right]
$$

Substituting Eq. (3.20) into Eq. (3.17), we get

$$
\begin{aligned}
& \int_{t_{0}}^{t_{f}^{*}}\left\{\frac{\partial F}{\partial x}-D_{t, t_{f}}^{\alpha} \frac{\partial F}{\partial D_{t_{0}, t}^{\alpha} x}-D_{t_{0}, t}^{\beta} \frac{\partial F}{\partial D_{t, t_{f}}^{\beta} x}\right\} \eta d t \\
& \quad+\left.\xi\left(t_{f}\right)\left(\frac{\partial F}{\partial D_{t_{0}, t}^{\alpha} x} D_{t_{0}, t}^{\alpha}(C-x)+\frac{\partial F}{\partial D_{t, t_{f}}^{\beta} x} D_{t, t_{f}}^{\beta}(C-x)+F\right)\right|_{t_{f}^{*}}=0 .
\end{aligned}
$$

Since $\eta(t)$ and $\xi\left(t_{f}\right)$ are arbitrary, it follows from a well-established result in the calculus of variations that

$$
\begin{aligned}
& \frac{\partial F}{\partial x}-D_{t, t_{f}}^{\alpha} \frac{\partial F}{\partial D_{t_{0}, t}^{\alpha} x}-D_{t_{0}, t}^{\beta} \frac{\partial F}{\partial D_{t, t_{f}}^{\beta} x}=0, \\
& \left.\left(\frac{\partial F}{\partial D_{t_{0}, t}^{\alpha} x} D_{t_{0}, t}^{\alpha}(C-x)+\frac{\partial F}{\partial D_{t, t_{f}}^{\beta} x} D_{t, t_{f}}^{\beta}(C-x)+F\right)\right|_{t_{f}}=0,
\end{aligned}
$$

where Eq. (3.22) is called fractional-order variational problem of Euler-Lagrange equation, and Eq. (3.23) is called terminal transversality condition. The proof is completed.

Remark 3.1 Note that for the fractional calculus of variation problems, the resulting Euler-Lagrange equation contains both LRLFD and RRLFD. This is expected since the optimum function must satisfy both terminal conditions. Further, for $\alpha=\beta=1$, we have $D_{t_{0}, t}^{\alpha}=d / d t$ and $D_{t, t_{f}}^{\beta}=-d / d t$. Then Eqs. (3.22)-(3.23) can be turned into the standard Euler-Lagrange equation and the terminal transversality condition

$$
\begin{aligned}
& \frac{\partial L}{\partial x}-\frac{d}{d t} \frac{\partial L}{\partial \dot{x}}=0, \\
& \left.\left((\dot{C}-\dot{x}) \frac{\partial F}{\partial \dot{x}}+F\right)\right|_{t=t_{f}}=0,
\end{aligned}
$$

where $\dot{x}=d x / d t$.

Remark 3.2 In control engineering, the majority target line is parallel to the $t$ axis, since $\dot{C}(t)=0$, hence

$$
\left.\left(F-D_{t, t_{f}}^{\alpha} x \frac{\partial F}{\partial D_{t_{0}, t}^{\alpha} x}-D_{t_{0}, t}^{\beta} x \frac{\partial F}{\partial D_{t, t_{f}}^{\beta} x}\right)\right|_{t=t_{f}}=0 .
$$

If $C(t)$ is perpendicular to the $t$ axis, since $\dot{C}(t)=\infty$, we get

$$
\left.\left(\frac{\partial F}{\partial D_{t_{0}, t}^{\alpha} x}+\frac{\partial F}{\partial D_{t, t_{f}}^{\beta} x}\right)\right|_{t=t_{f}}=0 .
$$


Remark 3.3 In a similar way, it is not difficult to infer that when the terminal is fixed, and the initial $c(t)$ is variable, the terminal transversality condition will change to

$$
\left.\left(F-\frac{\partial F}{\partial D_{t_{0}, t}^{\alpha} x} D_{t_{0}, t}^{\alpha}(x-c)-\frac{\partial F}{\partial D_{t, t_{f}}^{\beta} x} D_{t, t_{f}}^{\beta}(x-c)\right)\right|_{t=t_{0}}=0 .
$$

If $F(t)$ is parallel to the $t$ axis, we have

$$
\left.\left(F-\frac{\partial F}{\partial D_{t_{0}, t}^{\alpha} x} D_{t_{0}, t^{\alpha}}^{\alpha} x-\frac{\partial F}{\partial D_{t, t_{f}}^{\beta} x} D_{t, t_{f}}^{\beta} x\right)\right|_{t=t_{0}}=0 .
$$

If $F(t)$ is perpendicular to the $t$ axis, then

$$
\left.\left(\frac{\partial F}{\partial D_{t_{0}, t}^{\alpha} x}+\frac{\partial F}{\partial D_{t, t_{f}}^{\beta} x}\right)\right|_{t=t_{0}}=0 .
$$

Corollary 3.1 Let $J[x]=\int_{t_{0}}^{t_{f}} F\left(t, x, D_{t_{0}, t}^{\alpha_{1}} x, \ldots, D_{t_{0}, t}^{\alpha_{n}} x, D_{t, t_{f}}^{\beta_{1}} x, \ldots, D_{t, t_{f}}^{\beta_{m}} x\right) d t$ be a functional function satisfying the boundary conditions. Then a necessary condition for $J[x]$ to have an extremum for a given function $x(t)$ is that $x(t)$ satisfies the Euler-Lagrange equation and the terminal transversality condition:

$$
\begin{aligned}
& \frac{\partial F}{\partial x}-\sum_{j=1}^{n} D_{t, t_{f}}^{\alpha_{j}} \frac{\partial F}{\partial D_{t_{0}, t}^{\alpha_{j}} x}-\sum_{k=1}^{m} D_{t_{0}, t}^{\beta_{k}} \frac{\partial F}{\partial D_{t, t_{f}}^{\beta_{k}} x}=0 \\
& \left.\sum_{i=1}^{M}\left(\sum_{j=1}^{n} \frac{\partial F}{\partial D_{t_{0}, t}^{\alpha_{j}} x} D_{t_{0}, t}^{\alpha_{j}}\left(C_{i}-x\right)+\sum_{k=1}^{m} \frac{\partial F}{\partial D_{t, t_{f}}^{\beta_{k}} x} D_{t, t_{f}}^{\beta_{k}}\left(C_{i}-x\right)+F\right)\right|_{t=t_{f}}=0
\end{aligned}
$$

where $\alpha_{j} \in R^{+}(j=1, \ldots, n)$ and $\beta_{k} \in R^{+}(k=1, \ldots, m)$ are two sets of real numbers all greater than zero,

$$
\alpha_{\max }=\max \left(\alpha_{1}, \ldots, \alpha_{n}, \beta_{1}, \ldots, \beta_{m}\right)
$$

is the maximum of all these numbers, and $M$ is the integer such that $M-1 \leq \alpha_{\max }<M$. And $F$ is a function with continuous first and second (partial) derivatives with respect to all its arguments. Meanwhile, all functions $x(t)$ satisfy the following conditions:

$$
\begin{aligned}
& x(a)=x_{a 0}, \quad x^{(1)}(a)=x_{a 1}, \quad \ldots, \quad x^{(M-1)}(a)=x_{a(M-1)}, \\
& x\left(t_{f}\right)=C_{1}\left(t_{f}\right), \quad x^{(1)}\left(t_{f}\right)=C_{2}\left(t_{f}\right), \quad \ldots, \quad x^{(M-1)}\left(t_{f}\right)=C_{M}\left(t_{f}\right) .
\end{aligned}
$$

Corollary 3.2 Let $F\left(t, x_{1}, \ldots, x_{n}, y_{1}, \ldots, y_{2 n}\right)$ be a function with continuous first and second (partial) derivatives with respect to all its arguments. For $0<\alpha, \beta \leq 1$, consider the problem of finding necessary conditions for an extremum of a functional of the form

$$
J\left[x_{1}, \ldots, x_{n}\right]=\int_{t_{0}}^{t_{f}} F\left(t, x_{1}, \ldots, x_{n}, D_{t_{0}, t}^{\alpha} x_{1}, \ldots, D_{t_{0}, t}^{\alpha} x_{n}, D_{t, t_{f}}^{\beta} x_{1}, \ldots, D_{t, t_{f}}^{\beta} x_{n}\right) d t
$$


which depends on $n$ continuously differentiable functions $x_{1}(t), \ldots, x_{n}(t)$ satisfying the boundary conditions

$$
x_{j}\left(t_{0}\right)=x_{j 0}, \quad x_{j}\left(t_{f}\right)=C_{j}\left(t_{f}\right) \quad(j=1, \ldots, n) .
$$

Then, a necessary condition for the curve is

$$
x_{j}=x_{j}(t) \quad(j=1, \ldots, n),
$$

which satisfies the boundary conditions given by Eq. (3.38) to be an extremal of the functional given by Eq. (3.37), i.e., the functions $x_{j}(t)$ satisfy the following Euler-Lagrange equation and terminal transversality condition:

$$
\begin{aligned}
& \frac{\partial F}{\partial x_{j}}-D_{t, t_{f}}^{\alpha} \frac{\partial F}{\partial D_{t_{0}, t_{j}}^{\alpha} x_{j}}-D_{t_{0}, t}^{\beta} \frac{\partial F}{\partial D_{t, t_{f}}^{\beta} x_{j}}=0 \quad(j=1, \ldots, n), \\
& \left.\left(\frac{\partial F}{\partial D_{t_{0}, t_{j}}^{\alpha} x_{j}} D_{t_{0}, t}^{\alpha}\left(C_{j}-x_{j}\right)+\frac{\partial F}{\partial D_{t, t_{f}}^{\beta} x_{j}} D_{t, t_{f}}^{\beta}\left(C_{j}-x_{j}\right)+F\right)\right|_{t_{f}}=0 \quad(j=1, \ldots, n) .
\end{aligned}
$$

In vector notation, the above conditions can be written as

$$
\begin{aligned}
& \frac{\partial F}{\partial X}-D_{t, t_{f}}^{\alpha} \frac{\partial F}{\partial D_{t_{0}, t}^{\alpha} X}-D_{t_{0}, t}^{\beta} \frac{\partial F}{\partial D_{t, t_{f}}^{\beta} X}=0, \\
& \left.\left(\frac{\partial F}{\partial D_{t_{0}, t}^{\alpha} X} D_{t_{0}, t}^{\alpha}(C-X)+\frac{\partial F}{\partial D_{t, t_{f}}^{\beta} X} D_{t, t_{f}}^{\beta}(C-X)+F\right)\right|_{t_{f}}=0,
\end{aligned}
$$

where $X, C \in R^{n}$.

\section{Application}

In this section, we illustrate the importance of our results through one example.

Example 4.1 Consider the terminal variable fractional variational problem

$$
J(x)=\frac{1}{2} \int_{0}^{t_{f}}\left(D_{0, t}^{\alpha} x(t)\right)^{2} d t
$$

where $x(0)=0, x\left(t_{f}\right)=1-t_{f}$.

Proof We know that

$$
F=\frac{1}{2}\left(D_{0, t}^{\alpha} x(t)\right)^{2}
$$

and the fractional Euler-Lagrange equation

$$
D_{t, t_{f}}^{\alpha} D_{0, t}^{\alpha} x=0
$$

It can be shown that for $\alpha>\frac{1}{2}$, the solution is given as

$$
x(t)=(2 \alpha-1) \int_{0}^{t_{f}}\left[\left(t_{f}-\tau\right)(t-\tau)\right]^{\alpha-1} d \tau .
$$


Figure 1 Example 4.1.

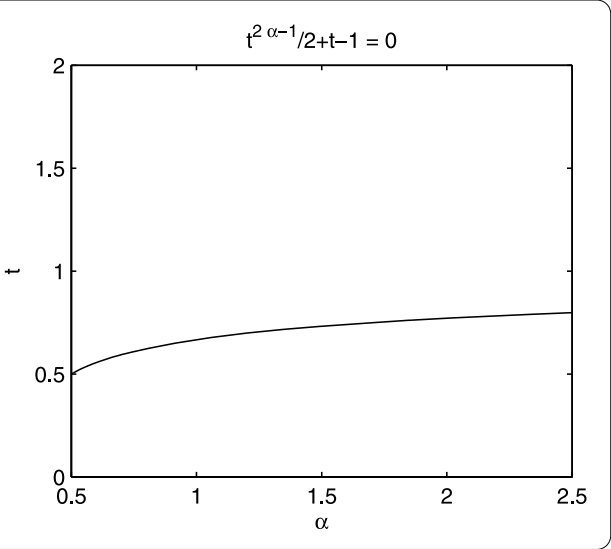

Now, we consider the transversality condition, by Eq. (3.4), we obtain

$$
\left\{\frac{1}{2}\left(D_{0, t}^{\alpha} x\right)^{2}+\left[D_{0, t}^{\alpha}(C(t)-x(t))\right] D_{0, t}^{\alpha} x\right\}_{t=t_{f}}=0
$$

By simplification, we get

$$
D_{0, t}^{\alpha} C(t)=\frac{1}{2} D_{0, t}^{\alpha} x(t)
$$

By Eqs. (4.4)-(4.6), $C(t)=1-t$ and the initial value, we obtain the optimal trajectory $\left(\alpha>\frac{1}{2}\right)$

$$
x^{*}(t)=\frac{(2 \alpha-1)}{2} \int_{0}^{t_{f}}\left[\left(t_{f}-\tau\right)(t-\tau)\right]^{\alpha-1} d \tau \text {. }
$$

Thus when $t=t_{f}$, we can get the optimal terminal times

$$
\frac{1}{2} t_{f}^{2 \alpha-1}=1-t_{f}
$$

By numerical simulation Eq. (4.8), we know that the equation has some solutions (see Figure 1).

This example with $\alpha=1$, for which the optimal trajectory is $x(t)=\frac{3}{2} t$, and then we have obtained the same result.

\section{Conclusions}

Necessary conditions for the optimality control of those systems are established. The case of piecewise continuous conditions and researching the minimum value of fractional differential equations principles will be considered in a future work. 


\section{Acknowledgements}

This article was supported by the Natural Science Foundation of China (Grant Nos. 11471015, 11371027), the natura Science Foundation of Anhui Province (No. 1508085MA01) and the Youth Superior Talent Key Foundation of Anhui Province (No. 2013SQRL142ZD). The authors would like to thank the referees for their valuable suggestions and comments.

Received: 16 January 2015 Accepted: 23 March 2015 Published online: 03 April 2015

\section{References}

1. Kilbas, AA, Srivastava, HM, Trujillo, JJ: Theory and Application of Fractional Differential Equations. Elsevier, New York (2006)

2. Oldham, KB, Spanier, J: The Fractional Calculus. Academic Press, New York (1974); renewed (2006)

3. Podlubny, I: Fractional Differential Equations. Academic Press, New York (1999)

4. Diethelm, K: The Analysis of Fractional Differential Equations. Springer, Berlin (2010)

5. Bhrawy, AH, Al-Zahrani, AA, Alhamed, YA, Baleanu, D: A new generalized Laguerre-Gauss collocation scheme for numerical solution of generalized fractional pantograph equations. Rom. Rep. Phys. 59(7-8), 646-657 (2014)

6. Doha, EH, Bhrawy, AH, Baleanu, D, Ezz-Eldien, SS, Hafez, RM: An efficient numerical scheme based on the shifted orthonormal Jacobi polynomials for solving fractional optimal control problems. Adv. Differ. Equ. 2015, 15 (2015). doi:10.1186/s13662-014-0344-z

7. Bhrawy, AH, Doha, EH, Baleanu, D, Ezz-Eldien, SS, Abdelkawy, MA: An accurate numerical technique for solving fractional optimal control problems. Proc. Rom. Acad., Ser. A : Math. Phys. Tech. Sci. Inf. Sci. 16(1), 47-54 (2015)

8. Bhrawy, AH, Zaky, MA: A method based on the Jacobi tau approximation for solving multi-term time-space fractional partial differential equations. J. Comput. Phys. 281, 876-895 (2015)

9. Ezz-Eldien, SS, Doha, EH, Baleanu, D, Bhrawy, AH: A numerical approach based on Legendre orthonormal polynomials for numerical solutions of fractional optimal control problems. J. Vib. Control (2015). doi:10.1177/1077546315573916

10. Jiao, F, Zhou, Y: Existence of solutions for a class of fractional boundary value problems via critical point theory. Comput. Math. Appl. 62, 1181-1199 (2011)

11. Agrawal, OP: Formulation of Euler-Lagrange equations for fractional variational problems. J. Math. Anal. Appl. 272 368-379 (2002)

12. Reiewe, F: Nonconservative Lagrangian and Hamiltonian mechanics. Phys. Rev. E 53, 1890-1899 (1996)

13. Reiewe, F: Mechanics with fractional derivatives. Phys. Rev. E 51, 5422-5428 (1997)

\section{Submit your manuscript to a SpringerOpen ${ }^{\ominus}$ journal and benefit from:}

- Convenient online submission

- Rigorous peer review

- Immediate publication on acceptance

- Open access: articles freely available online

- High visibility within the field

- Retaining the copyright to your article 